\title{
A prion-like domain in ELF3 functions as a thermosensor in Arabidopsis
}

\author{
Authors: \\ Jae-Hoon Jung ${ }^{1,2 *}$, Antonio D. Barbosa ${ }^{1 *}$, Stephanie Hutin ${ }^{4 *}$, Janet R. Kumita ${ }^{3}$, \\ Mingjun Gao ${ }^{1}$, Dorothee Derwort ${ }^{1}$, Catarina S. Silva ${ }^{4}$, Xuelei Lai ${ }^{1,4}$, Elodie Pierre ${ }^{4}$, \\ Feng Geng ${ }^{1}$, Sol-Bi Kim², Sujeong Baek ${ }^{2}$, Chloe Zubieta ${ }^{4}$, Katja E. Jaeger ${ }^{1,5}$ and \\ Philip A. Wigge ${ }^{1,5,6}$
}

${ }^{1}$ Sainsbury Laboratory, University of Cambridge, Cambridge, CB2 1LR, United Kingdom. ${ }^{2}$ Department of Biological Sciences, Sungkyunkwan University, Suwon 16419, South Korea.

${ }^{3}$ Department of Chemistry, University of Cambridge, Lensfield Rd, Cambridge CB2 1EW, United Kingdom.

${ }^{4}$ Laboratoire de Physiologie Cellulaire and Végétale, Université Grenoble Alpes/CNRS/CEA/INRAE, 17 Rue des Martyrs, 38054 Grenoble, France.

5Leibniz-Institut für Gemüse- und Zierpflanzenbau, Theodor-Echtermeyer-Weg 1, 14979 Großbeeren, Germany.

${ }^{6}$ Institute of Biochemistry and Biology, University of Potsdam, 14476 Potsdam, Germany.

${ }^{*}$ These authors contributed equally

§Corresponding author: wigge@igzev.de

------------(Introductory paragraph, 271 words)

Temperature is a major environmental variable governing plant growth and development, and climate change has already altered the phenology of wildplants and crops ${ }^{1}$. However, the mechanisms by which plants sense temperature are not well understood. Environmental signals, including temperature, are integrated into growth and developmental pathways via the circadian clock and the activity of the Evening Complex (EC), a major signalling hub and core clock component ${ }^{2,3}$. The EC acts as a temperature responsive transcriptional repressor, providing rhythmicity and temperature responsiveness to growth via unknown mechanisms ${ }^{2,4-6}$. The EC consists of EARLY FLOWERING3 (ELF3) ${ }^{4,7}$, a large scaffold protein and key component 
in temperature sensing, ELF4, a small alpha helical protein, and LUX ARRYTHMO (LUX), a DNA binding protein required for recruitment of the EC to transcriptional targets. ELF3 contains a polyglutamine (polyQ) repeat ${ }^{8-10}$, embedded within a predicted prion domain $(\operatorname{PrD})$. We find the length of the polyQ repeat correlates with thermal responsiveness. ELF3 in plants from hotter climates, which have no detectable PrD domain, is active at high temperature and these plants lack thermal responsiveness. ELF3 temperature sensitivity is also modulated by the levels of ELF4, indicating that ELF4 can stabilise ELF3 function. In both Arabidopsis and a heterologous system, ELF3-GFP forms speckles within minutes in response to higher temperatures in a PrD-dependent manner. This suggests that ELF3 is thermosensory. A purified fragment encompassing the ELF3 PrD reversibly forms liquid droplets in response to temperature in vitro, indicating that these properties reflect a direct biophysical response conferred by the $\operatorname{PrD}$. The ability of temperature to rapidly convert ELF3 between active and inactive states via phase transition represents a novel thermosensory mechanism.

-(Main text, 1605 words)

Arabidopsis ELF3 contains a polyglutamine (polyQ) repeat which varies from 7 to 29 residues in length in natural populations, and has been associated with phenotypic variation ${ }^{8-10}$ (Fig. 1a). We therefore investigated if the length of the polyQ repeat influences ELF3 activity. We find that in an isogenic Col-0 background, complementing elf3-1 with ELF3 transgenes encoding increasing polyQ lengths results in increased sensitivity to warm temperature as measured by hypocotyl elongation (Fig. 1b, Extended Data Fig. 1, Extended Data Fig. 14). The effects of altering polyQ length are mild, in agreement with other studies ${ }^{8,11}$, and Q0 lines are still thermally responsive. This indicated that additional features of ELF3 are also required for responding to temperature. Since the polyQ repeat is located in the centre of a region predicted to be a prion domain ${ }^{12}$ (PrD; residues 430-609) (Fig. 1a), we hypothesized that this might confer temperature responsiveness. If the PrD of ELF3 plays a role in temperature responsiveness in Arabidopsis, we wondered if it varies in plants adapted to different climates. Indeed, ELF3 from Solanum tuberosum and Brachypodium distachyon are not predicted to have 
PrD regions (Fig. 1a; Extended Data Fig. 2). Since accelerated flowering is a major adaptive response of Arabidopsis to warm temperature, we investigated if StELF3 and BdELF3 alter this trait. BdELF3 and StELF3 are functional in Arabidopsis and complement elf3-1 (Extended Data Fig. 3). At $22{ }^{\circ} \mathrm{C}$ these plants resemble wild-type, however at $27^{\circ} \mathrm{C}$ they lose almost all their thermally responsive early flowering (Fig. 1c). This shows that these versions of ELF3 lacking a PrD are largely unable to respond to warm temperature. To test whether the thermal responsiveness of Arabidopsis ELF3 is due to the PrD itself, we created a chimeric version, where we replaced the PrD of Arabidopsis with the corresponding sequence of BdELF3 (Extended Data Fig. 2). Chimeric ELF3-BdPrD shows a suppression of temperature responsive flowering, confirming that the PrD from Arabidopsis confers thermal responsiveness (Fig. 1c).

The activity of ELF3 is modulated by binding to the small peptide ELF4 ${ }^{13}$. To further understand if ELF4 is contributing to the thermal responsiveness of ELF3, we investigated the effect of temperature on hypocotyl elongation and flowering time in elf4-101 and elf4-2. At 22 and $27^{\circ} \mathrm{C}$, elf4 alleles largely resemble elf3-1, consistent with the key role for ELF4 in the $\mathrm{EC}^{2,13}$. At $17^{\circ} \mathrm{C}$, ELF4 becomes dispensable for controlling both hypocotyl elongation and flowering time and elf4-101 and elf4-2 have similar phenotypes to Col-0 (Fig. 2a). These results suggest that ELF4 plays a role in buffering the temperature responsiveness of ELF3 at higher temperatures, leading us to hypothesize that overexpressing ELF4 may stabilize ELF3, as is suggested by in vitro studies ${ }^{15}$. ELF4 expression is circadianly regulated, peaking at the end of the day and rapidly declining during the night ${ }^{14}$. We observe that plants constitutively overexpressing ELF4 are largely unable to respond to temperature, as seen by both hypocotyl elongation and flowering time (Fig. $2 b)$, indicating that the presence of higher levels of ELF4 is sufficient to maintain ELF3 in the active state even at $27^{\circ} \mathrm{C}$ (Fig. 2b). This appears to be a consequence of modulating ELF3 function, since overexpressing ELF4 has no detectable effect in the elf3-1 background, and ELF3 overexpression does not change thermal responsiveness (Extended Data Fig. 4). We mapped the 
domain of ELF3 that interacts with ELF4 to a low complexity region adjacent to the PrD (Extended Data Fig. 11).

Since ELF3 is a temperature dependent transcriptional repressor, we sought to determine if the phenotypic variation in responsiveness to temperature can be accounted for by variation in occupancy of ELF3 on target genes. As shown previously ${ }^{3,4}$, the occupancy of ELF3 on target genes decreases with warmer temperature (Fig. 2c, Extended Data Fig. 5). Consistent with our phenotypic observations, forms of ELF3 lacking a PrD also lose their temperature responsiveness of binding, indicating that the $\operatorname{PrD}$ directly modulates the thermoresponsiveness of ELF3 binding at target genes (Fig. 2d, Extended Data Fig. 4). Finally, overexpressing ELF4 is also sufficient to stabilize ELF3 binding and abolish the temperature response (Fig. 2e, Extended Data Fig. 5).

Since ELF3 functions as a transcriptional regulator we sought to determine if our observed changes in occupancy have a detectable influence on the ELF3dependent transcriptome. To identify ELF3-dependent genes, we searched for genes showing a pattern of expression similar to $L U X$, i.e. having a strong up-regulation in elf3-1 and a reduced expression in ELF3-OE at ZT8 and ZT12. In this way we were able to identify 325 genes whose expression is dependent on the level of ELF3 activity, which includes key EC targets such as PIF4 and GI. These genes are less thermally responsive in backgrounds expressing BdELF3 or overexpressing ELF4 (Extended Data Fig. 6, 7 and 8). We observe a mild effect of the polyQ lines on the expression of ELF3dependent genes, consistent with the more subtle phenotypes for hypocotyl length we observe for these lines (Extended Data Fig. 6, 7). We find 112 genes associated with the strongest ELF3 ChIP-seq peaks, and 25 of these are common with the 325 ELF3-dependent genes, consistent with this being a direct mechanism (Extended Data Fig. 8). The ELF3-dependent genes directly bound by ELF3 show a clear temperature responsiveness in their expression, and this is affected when ELF3 binding is stabilized. 
To investigate if temperature may directly control ELF3 activity, we analyzed the behaviour of natively expressed ELF3-GFP in planta. At $17^{\circ} \mathrm{C}$, ELF3-GFP is nuclear with a diffuse signal. Upon shifting to $35^{\circ} \mathrm{C}$, we see multiple bright speckles form, a behaviour that is specific to the presence of the $\operatorname{PrD}$, since the chimeric ELF3 with the BdPrD remains largely diffuse in response to warmer temperature (Fig. 3a). This behaviour is also observed at $27^{\circ} \mathrm{C}$ (Fig. $3 b)$. Increasing polyQ length also results in a greater tendency to form speckles (Extended Data Fig. 9). Since ELF3 in planta may be influenced by other factors that have co-evolved to control its activity, we sought to determine if ELF3 expressed in Saccharomyces cerevisiae, a heterologous system lacking ELF3 related genes, is temperature responsive. Under a low expression system, ELF3-GFP forms a largely diffuse signal in yeast cells, while when highly expressed, it forms bright puncta or speckles (Fig. 3c). We next investigated the influence of temperature on ELF3-GFP in yeast. At 19 ${ }^{\circ} \mathrm{C}$, the signal is largely diffuse. Shifting cells to $35^{\circ} \mathrm{C}$ results in a rapid formation of sharp punctate GFP signals, that is more significant for ELF3 Q7 and Q35 compared to ELF3 BdPrD or free GFP (Fig. 3d, e). These effects appear specific for ELF3, since $35^{\circ} \mathrm{C}$ is below the temperature required for endogenous proteins to aggregate ${ }^{16}$, and we observe robust cell growth and protein expression under these conditions (Extended Data. Fig. 10). While classically prions are associated with stable insoluble aggregates in the cell, the EC and ELF3 undergo diurnal cycles of activity and temperatures fluctuate over short timescales, suggesting reversibility of the temperature response is likely important. Indeed, the EC rapidly returns to full activity when plants are shifted from 27 to $22^{\circ} \mathrm{C}^{4}$, leading us to hypothesize that the formation of speckles may be reversible. This is the case in yeast, as returning cells from 35 to $19^{\circ} \mathrm{C}$ results in a rapid reduction in the number of speckles (Fig. 3f, g).

The results so far are consistent with ELF3 being able to adopt two conformations: an active soluble form, and, at higher temperatures, a higher order multimeric form that is visible as bright speckles. It has been suggested that a major biological function of prion-like proteins is to act as environmental switches, as they are able to rapidly change conformation and form liquid droplets ${ }^{17}$. While ELF3 is largely insoluble in vitro, we identified a soluble 
peptide fragment spanning the PrD (ELF3 PrD, residues 388-625). AtELF3 $\operatorname{PrD}$, in contrast to BdELF3 PrD, rapidly and reversibly forms liquid droplets as a function of ionic strength, protein concentration and temperature (Fig. 4a, b, Extended Data 12). To analyze the dynamics of this behaviour, we purified ELF3 PrD fused to GFP (PrD-GFP). Decreasing the salt concentration and pH induces PrD-GFP to undergo a phase transition, forming micron sized spherical droplets. The droplets are highly mobile in solution and are able to fuse, indicative of phase separated liquids (Fig. 4c and Extended Data Fig. 13a, b). Using fluorescence recovery after photobleaching (FRAP) we see that recovery fractions range from 0.1 to 0.8 with recovery half-lives from seconds to minutes (Fig. 4d and Extended Data Fig. 13c, d). To determine if the $\operatorname{PrD}$ is thermoresponsive, we analyzed liquid droplet formation as a function of temperature. The purified ELF3 PrD peptide is more soluble at low temperature, but undergoes a sharp phase transition with a midpoint at 28.7 $\pm 1.8^{\circ} \mathrm{C}$ under our conditions (Fig. 4 e). Upon decreasing the temperature, this process is significantly reversible and the cycle can be repeated (Fig. 4f). This agrees with the temperature responsiveness of the ELF3 system in Arabidopsis seedlings, where the strongest phenotypic effects are observed at $27^{\circ} \mathrm{C}$. This response is specific for the $\operatorname{PrD}$, since the equivalent peptide fragment of BdELF3 shows no liquid droplet formation (Fig. 4e). These results indicate that the PrD domain of ELF3 serves as a tunable thermosensor. Intrinsically disordered proteins frequently display thermoresponsive liquidliquid phase separation, a behaviour driven by solvent-mediated interactions in a sequence dependent manner ${ }^{18}$. Since PrD and intrinsically disordered protein sequences are widespread within eukaryotes ${ }^{19}$, it will be interesting to see if they have been recruited to provide thermosensory behaviour via phase transitions in other signalling contexts, such as the reversible aggregation of proteins in response to heat stress in yeast ${ }^{16}$.

(End of main text)

We thank M. Perutz for discussions on polyQ proteins. We thank the microscopy facility MuLife of IRIG/DBSCl, funded by CEA Nanobio and labex Gral for equipment access and use and Laëtitia Kurzawa and Fabrice Senger for technical assistance and helpful discussions. This work used the platforms 
of the Grenoble Instruct-ERIC Center (ISBG : UMS 3518 CNRS-CEA-UGAEMBL) with support from FRISBI (ANR-10-INBS-05-02) and Labex GRAL (ANR-CBH-EUR-GS) within the Grenoble Partnership for Structural Biology (PSB). PW and CZ received funding from Instruct-ERIC (PID: 2236). This work has supported by the National Research Foundation of Korea(NRF) grant funded by the Korea government(MSIT)(No. 2019R1C1C1010507 to J.H.J). This work was funded by ANR-19-CE20-0021-01 PRC (CZ). PAW receives funding from the Leibniz Foundation.

\section{Data Availability Statement:}

Sequencing data for gene expression analysis (RNA-seq) and protein-DNA interactions (ChIP-seq) have been deposited in the publicly available databasej GEO, Accession Code GSE137264, with review access token "mjqdmmamvtsdret".

\section{Code Availability:}

The code to produce the figures from the processed files is available at https://github.com/shouldsee/polyq-figures . To enable easier browsing, a static site is hosted at https://shouldsee.github.io/polyq-figures . The inhouse pipeline for mapping is available at https://github.com/shouldsee/synoBio .

\section{Author contributions:}

JHJ, ADB, SH, JRK, CZ, KEJ and PAW conceived the study and wrote the manuscript. JHJ, MG generated transgenic plants and mutants and analysed their phenotypes. ADB performed in vivo imaging experiments in yeasts and plants. SH, JRK, CSS, XL, EP, CZ performed and analysed in vitro phase separation assays. KEJ performed ChIP- and RNA-seq experiments. DD and FG analysed sequencing data. SBK and SB performed yeast two hybrid assays and analysed gene expression levels in transgenic plants. CZ, KEJ and PAW supervised experimental work.

\section{References:}

1. Scheffers, B. R. et al. The broad footprint of climate change from genes to biomes to people. Science (80-. ). 354, aaf7671 (2016).

2. Nusinow, D. A. et al. The ELF4-ELF3-LUX complex links the circadian clock to diurnal control of hypocotyl growth. Nature 475, 398-402 (2011).

3. Ezer, D. et al. The evening complex coordinates environmental and endogenous signals in Arabidopsis. Nat. Plants 3, 17087 (2017).

4. Box, M. S. et al. ELF3 controls thermoresponsive growth in Arabidopsis. 
Curr. Biol. 25, 194-9 (2015).

5. Mizuno, T. et al. Ambient Temperature Signal Feeds into the Circadian Clock Transcriptional Circuitry Through the EC Night-Time Repressor in Arabidopsis thaliana. Plant Cell Physiol. 0, 1-19 (2014).

6. Raschke, A. et al. Natural variants of ELF3 affect thermomorphogenesis by transcriptionally modulating PIF4-dependent auxin response genes. BMC Plant Biol. 15, 197 (2015).

7. Nieto, C., Lopez-Salmeron, V., Daviere, J. \& Prat, S. ELF3-PIF4 interaction regulates plant growth independently of the Evening Complex. Curr. Biol. 25, 187-193 (2015).

8. Undurraga, S. F. et al. Background-dependent effects of polyglutamine variation in the Arabidopsis thaliana gene ELF3. Proc. Natl. Acad. Sci. U. S. A. 109, 19363-7 (2012).

9. Tajima, T., Oda, A., Nakagawa, M., Kamada, H. \& Mizoguchi, T. Natural variation of polyglutamine repeats of a circadian clock gene ELF3 in Arabidopsis. Plant Biotechnol 24, 237-240 (2007).

10. Jiménez-Gómez, J. M., Wallace, A. D. \& Maloof, J. N. Network analysis identifies ELF3 as a QTL for the shade avoidance response in arabidopsis. PLoS Genet. 6, (2010).

11. Press, M. O. \& Queitsch, C. Variability in a short tandem repeat mediates complex epistatic interactions in Arabidopsis thaliana. Genetics (2017). doi:10.1534/genetics.116.193359

12. Lancaster, A. K., Nutter-Upham, A., Lindquist, S. \& King, O. D. PLAAC: a web and command-line application to identify proteins with prion-like amino acid composition. Bioinformatics 30, 2501-2502 (2014).

13. Herrero, E. et al. EARLY FLOWERING4 recruitment of EARLY FLOWERING3 in the nucleus sustains the Arabidopsis circadian clock. Plant Cell 24, 428-43 (2012).

14. Doyle, M. R. et al. The ELF4 gene controls circadian rhythms and flowering time in Arabidopsis thaliana. Nature 419, 74-77 (2002).

15. Silva, C. S. et al. Molecular mechanisms of Evening Complex activity in Arabidopsis. Proc. Natl. Acad. Sci. XX, XX (2020).

16. Wallace, E. W. J. et al. Reversible, Specific, Active Aggregates of Endogenous Proteins Assemble upon Heat Stress. Cell (2015). 
doi:10.1016/j.cell.2015.08.041

17. Franzmann, T. M. et al. Phase separation of a yeast prion protein promotes cellular fitness. Science 359, eaao5654 (2018).

18. Dignon, G. L., Zheng, W., Kim, Y. C. \& Mittal, J. TemperatureControlled Liquid-Liquid Phase Separation of Disordered Proteins. ACS Cent. Sci. acscentsci.9b00102 (2019). doi:10.1021/acscentsci.9b00102

19. Si, K. Prions: What Are They Good For? Annu. Rev. Cell Dev. Biol. 31, 149-169 (2015).

\section{FIGURE LEGENDS}

Fig. 1: The polyQ repeat of ELF3 is embedded within a predicted priondomain which is essential for thermal responsiveness.

a, Arabidopsis ELF3 (AtELF3) contains a polyQ repeat embedded with a predicted prion-domain (PrD), while this PrD signature is absent in ELF3 from Brachypodium distachyon (BdELF3) and Solanum tuberosum (SdELF3). Prion domains predicted using $\mathrm{PLAAC}^{12}$. b, At $17^{\circ} \mathrm{C}, E L F 3$ is required to prevent hypocotyl elongation, but different polyQ lengths in ELF3 do not perturb ELF3 function. At $27^{\circ} \mathrm{C}$, the responsiveness of ELF3 to temperature increases with the length of the polyQ. (Naming convention: Q7-17, 7 refers to length of polyQ repeat, and 17 is the individual transgenic line used in the study). c, The ELF3 PrD plays a critical role in the thermal control of flowering time. Overexpressing AtELF3 does not change the thermal induction of flowering, indicating that simply increasing ELF3 protein level is not sufficient to delay flowering, while overexpressing BdELF3 causes an almost complete loss of thermal induction, and StELF3 has a milder influence. These effects are also apparent when BdELF3 is expressed using the native ELF3 promoter, and dependent on the $\operatorname{PrD}$, since replacing this domain of AtELF3 with the corresponding $\mathrm{Bd}$ sequence is sufficient to greatly reduce the thermal induction of flowering.

Fig. 2: ELF4 is required to stabilize the activity of the EC at warmer temperatures.

a, At lower temperatures, ELF4 becomes dispensable for controlling both hypocotyl elongation and flowering, but with increasing temperature, ELF4 
assumes a greater role. b, ELF4 overexpression reduces thermal responsiveness of hypocotyl elongation and markedly decreases the flowering time response to temperature, and this response is entirely dependent on ELF3. c, ELF3 binding at targets, which is measured by ChIP-seq, is temperature dependent and declines genome-wide at $27^{\circ} \mathrm{C}$. d, Transgenic plants with stabilized forms of ELF3 do not respond to temperature. e, ELF4 overexpression stabilizes ELF3 and removes its temperature responsiveness of binding to targets. Scale bars, $5 \mathrm{~mm}(\mathbf{a}, \mathbf{b})$

Fig. 3: High temperature induces the formation of reversible ELF3 speckles in vivo.

a, Seedlings expressing GFP-tagged ELF3 (Q7) or a chimeric ELF3 where the PrD has been replaced by the corresponding region of Brachypodium ELF3 (BdPrD) grown in short photoperiods for 7 days at $17^{\circ} \mathrm{C}$. Roots imaged by confocal microscopy before and after incubation at $35^{\circ} \mathrm{C}$ for $15 \mathrm{~min}$. b. Seedlings as in (a) but shifted to $27^{\circ} \mathrm{C}$ for $2 \mathrm{~h}$. C, S. cerevisiae cells expressing GFP-tagged ELF3 (Q7) from a centromeric plasmid (Low) or an episomal plasmid (High), to achieve low or high expression levels of the protein, were grown in selective synthetic complete medium at $30^{\circ} \mathrm{C}$; Sec63mCherry was used as an ER reporter. d, Quantification of ELF3 speckles in S. cerevisiae cells expressing free GFP (GFP only), ELF3 (Q7), ELF3 with a longer polyQ repeat (Q35), or ELF3 with the $\operatorname{PrD}$ of $B$. distachyon (BdPrD) grown overnight at $19^{\circ} \mathrm{C}$ and incubated at the indicated temperatures for 30 min. e, representative images of cells from d at $35^{\circ} \mathrm{C}$. f, S. cerevisiae cells expressing ELF3 were grown at $19^{\circ} \mathrm{C}$ overnight and incubated at $35^{\circ} \mathrm{C}$ for 30 $\min (19>35)$ followed by incubation at $19^{\circ} \mathrm{C}$ for $60 \min (35>19)$. $\mathbf{g}$, Quantification of ELF3 speckles for cells in f. Scale bars, $40 \mu \mathrm{m}$ ( $\mathrm{a}$ and b) and $5 \mu \mathrm{m}$ (a and b inset, $c, e$, and $f),{ }^{* *} p<0.01$.

Fig. 4: The PrD of ELF3 undergoes a reversible phase transition in response to temperature.

a, Purified ELF3 PrD peptide forms liquid droplets at $27^{\circ} \mathrm{C}$ in vitro. b, The equivalent protein domain from BdELF3, which is not predicted to contain a 
$\operatorname{PrD}$, remains soluble and does not show any liquid droplet formation $\mathbf{c}$, Purified ELF3 PrD-GFP protein forms spherical droplets in vitro, which fuse. d, ELF3 PrD-GFP droplets show rapid recovery after photobleaching, indicating they are liquid droplets. e, Light scattering assay as a function of temperature for ELF3 PrD (15 $\mu \mathrm{M}$; black circle), BdELF3 (15 $\mu \mathrm{M}$; grey circle) and buffer alone (50 mM CAPS pH 9.7, 150 mM NaCl, 1 mM TCEP; open triangles). Dashed line shows curve-fitting using a 4-parameter sigmoidal equation. The $T_{m}$ for ELF3 $\operatorname{PrD}$ is $28.7 \pm 1.8^{\circ} \mathrm{C}$ and the spectrum is representative of three independent experiments. $\mathbf{f}$, Reversibility of light scattering as a function of temperature for ELF3 PrD (15 $\mu \mathrm{M} ; 50$ mM CAPS pH 9.7, $200 \mathrm{mM} \mathrm{NaCl}, 1 \mathrm{mM}$ TCEP). On the same sample, the temperature was increased and decreased three times in succession $\left(1^{\circ} \mathrm{C} / \mathrm{min}\right)$. The observed turbidity was reversible and consistently returned to $\mathrm{Abs}_{440 \mathrm{~nm}}=$ $0.432 \pm 0.02$. Interestingly, the initial absorbance reading for repeat 3 $\left(\mathrm{Ab}_{440 \mathrm{~nm}}=0.288\right)$ is lower than for repeat $2\left(\mathrm{Abs}_{440 \mathrm{~nm}}=0.373\right)$ and this is likely due to time dependent equilibration (as noted in Extended Figure 12c). .The spectra are representative of two independent experiments and similar results were observed for samples at $5 \mu \mathrm{M}$. Scale bars, $50 \mu \mathrm{m}(\mathbf{a}, \mathbf{b})$ and $5 \mu \mathrm{m}(\mathbf{c}, \mathbf{d})$.

\section{EXTENDED DATA FIGURE LEGENDS}

Extended Data Fig. 1 | The length of the polyQ repeat within the ELF3 PrD influences temperature responsiveness.

Hypocotyl length of transgenic plants with altered polyQ lengths at different temperatures. Data for 17 and $27^{\circ} \mathrm{C}$ are used in Fig. $\mathbf{1 b}$.

\section{Extended Data Fig. 2 | Multiple sequence alignment of ELF3 proteins} generated with ClustalW. ELF3 amino acid sequences from three different plant species were included in the alignment. The region indicated by an arrow were used to create a chimeric version of Arabidopsis ELF3, where the ELF3 PrD was replaced with the corresponding sequence of BdELF3 or StELF3. 
Extended Data Fig. 3 | Plants expressing ELF3 lacking a detectable PrD from potato (St) and Brachypodium (Bd) rescue elf3-1 at $22^{\circ} \mathrm{C}$ and show reduced $F T$ expression.

$\mathbf{A}$, and $\mathbf{b}$. Transgenic plants in the elf3-1 background expressing different forms of ELF3 expressed constitutively (35S promoter) or under the endogenous AtELF3 promoter (ELF3 pro) grown in SD conditions until bolting. c, Relative expression of $F T$ at zeitgeber time (ZT) 8 was analyzed by RTqPCR. Twelve-day-old seedlings grown at different temperatures under short photoperiod conditions (SDs) were used to analyze transcript accumulation. Bars indicate standard error of the mean.

Extended Data Fig. 4 | ELF4 modulates the temperature responsiveness of EC activity.

At low temperatures, ELF4 is dispensable, and elf4-2 mutants have similar hypocotyl phenotypes to wild-type plants. As temperature increases, the role of ELF4 becomes increasingly important, as measured by hypocotyl length. Overexpressing ELF3 (ELF3-OE) is not sufficient to change thermal responsiveness and ELF3 overexpression has no effect in the elf4-2 background at $27^{\circ} \mathrm{C}$, indicating that ELF4 plays an important role at higher temperatures.

Extended Data Fig. 5 | The binding of ELF3 at target genes depends on temperature, and stabilized forms of ELF3 are less temperature responsive than wild-type ELF3.

Average ELF3 ChIP-seq peak signals are measured as fold-enrichment over input (as calculated by MACS2) across multiple transgenic lines expressing different ELF3 variants.

Extended Data Fig. 6 | The expression of ELF3 dependent genes is influenced by temperature and the PrD of ELF3.

325 transcripts having ELF3 dependent expression were analyzed in RNAseq datasets for different genotypes at 22 and $27^{\circ} \mathrm{C}$. As expected, gene expression is generally suppressed at $22^{\circ} \mathrm{C}$ (red), apart from in the case of the elf3-1 background, where genes are up-regulated (green). Lines 
overexpressing $B d E L F 3$ show less activation at $27^{\circ} \mathrm{C}$, consistent with their later flowering phenotypes. Just replacing the Arabidopsis PrD with the corresponding region from BdELF3 (in the ELF3pro::BdELF3 at $27^{\circ} \mathrm{C}$ ) is sufficient to greatly reduce the upregulation of ELF3 dependent genes at 27 ${ }^{\circ} \mathrm{C}$. Up-regulation of ELF3 dependent genes also occurs in an elf3-1 mutant when ELF4 is overexpressed, consistent with ELF3 being necessary for ELF4 action.

Extended Data Fig. 7 | The expression of ELF3 dependent genes is influenced by temperature and the polyQ repeat of ELF3.

325 transcripts having ELF3 dependent expression were used analyzed in RNA-seq datasets for different genotypes at 22 and $27^{\circ} \mathrm{C}$. Plants expressing ELF3 with a truncated polyQ repeat (ELF3-Q0) show a reduced expression of $E L F 3$ dependent genes at $27^{\circ} \mathrm{C}$, consistent with their shorter hypocotyl phenotype.

Extended Data Fig. 8 | ELF3 target genes show altered responsiveness to temperature in backgrounds where stabilized versions of ELF3 are expressed.

Heat map showing that ELF3 bound targets that are usually induced by shifting to $27^{\circ} \mathrm{C}$ (green) become less temperature responsive in backgrounds where ELF3 is more stable.

\section{Extended Data Fig. 9 | The length of the polyQ repeat within the ELF3} PrD influences speckle formation by temperature in vivo.

a, Arabidopsis seedlings expressing GFP-tagged ELF3 variants with either no poly $Q$ repeat $(Q 0)$, the $W T$ poly $Q(Q 7)$, a poly $Q$ with 20 or 30 glutamines $(Q 20$ and $\mathrm{Q} 30$, respectively), or the $\operatorname{PrD}$ replaced by the corresponding region from Brachypodium distachyon ELF3 (BdPrD). Seedlings were grown in short photoperiods for 7 days at $17^{\circ} \mathrm{C}$. Roots were imaged by confocal microscopy before and after incubation at $30^{\circ} \mathrm{C}$ for $15 \mathrm{~min}$. b, Quantification of the degree of speckle formation in (a). Regions of the roots corresponding to the size of individual cells were selected, and the mean, standard deviation (sd) and maximum (max) gray value were measured in ImageJ. It was assumed that 
speckle formation would lead to higher gray values and higher frequency of speckles within the analysis region would increase the standard deviation. c, Relative FT expression in the ELF3pro::ELF3-GFP transgenic plants examined. Relative expression of $F T$ at zeitgeber time (ZT) 8 was analyzed by RT-qPCR. Twelve-day-old seedlings grown at different temperatures under SDs were used to analyze transcript accumulation. Bars indicate standard error of the mean. Warm temperature effect on the induction of $F T$ was not observed only in the transgenic plants containing ELF3 variants with the BdPrD. Scale bar, $40 \mu \mathrm{m}(\mathbf{a})$.

\section{Extended Data Fig. 10 | Yeast strains show no growth defect after} incubation at the indicated temperatures used in the speckle formation experiments and express detectable levels of ELF3-GFP

a, Temperature shifts do not affect yeast viability. Yeast cells were grown overnight at $19^{\circ} \mathrm{C}$ and shifted to the indicated temperatures for $30 \mathrm{~min}$ (as done in temperature shifts used for speckle inductions). Serial dilutions were spotted onto YPD plates and incubated at $30^{\circ} \mathrm{C}$ for the indicated times.

b, Yeast cells expressing the indicated ELF3-GFP constructs or an empty vector were grown overnight in selective media to exponential phase at $30^{\circ} \mathrm{C}$. Cells (approximately 9 OD600) were pelleted, washed with sterile water, and lysed in $100 \mu \mathrm{l}$ SDS-sample buffer with $0.5 \mathrm{~mm}$ diameter glass beads (BioSpec Products, Bartlesville, OK) by two rounds of boiling for $2 \mathrm{~min}$ and vortexing for $30 \mathrm{sec}$. Protein extracts were centrifuged at 13,000 rpm for 15 min, and the supernatants analysed by Western blot using anti-GFP antibody (a gift from A. Peden). Western blot signals were developed using ECL (GE Healthcare, Little Chalfont UK).

\section{Extended Data Fig. 11 | ELF3-ELF4 protein-protein interactions in yeast cells}

a, ELF3 constructs used. Numbers indicate reside positions. PrD, prionrelated domain. The domain structure of the ELF3 protein was determined using SMART protein domain annotation resource (http://smart.embl.de). ELF3 does not contain any specific domains except for low complexity regions, which are regions in protein sequences that differ from the 
composition and complexity of most proteins with normal globular structure. $\mathbf{b}$, Interactions of ELF3 with ELF4 in yeast cells. Cell growth on selective media was examined. Please note that the ELF3 region containing a low complexity region, which is not overlapped with $\operatorname{PrD}$ sequence, is responsible for the interaction with ELF4. A soluble form of ELF3 peptide, which is used for in vitro experiments, does not include the region required for the interaction with ELF4.

\section{Extended Data Fig. 12 | ELF3 PrD proteins show phase change characteristics in vitro.}

a, SDS gel (12 \% polyacrylamide) for ELF3 peptides for BdELF3 PrD, ELF3 PrD, and ELF3 PrD-GFP. M, molecular weight marker. Proteins were expressed and purified at least ten times with highly reproducible results. $\mathbf{b}$, Phase diagram of ELF3 PrD peptide with respect to salt and protein concentration. Examples of phases are shown in the right panel. c, Droplet formation is dynamic with droplets re-entering the soluble phase over time as measured from two independent samples (mean shown) by changes in $\mathrm{A}_{340}$ after droplet formation induced by dilution from a high salt to low salt buffer (50 mM CAPS, pH 9.7, $1 \mathrm{mM}$ TCEP, 500 to $150 \mathrm{mM} \mathrm{NaCl}$ ).

\section{Extended Data Fig. 13 | ELF3 PrD droplets fuse.}

a, Fusion of two droplets over time with intensity profiles of each droplet shown below the images. b, Fusion of ELF3 PrD droplets. Two examples are shown. c, Example of photobleaching and recovery over time. Images were taken (left to right) before, after and at time points 30 s, 120 s, 240 s postphotobleaching. d, FRAP recovery curves for $\mathbf{c}$ and mean $+/-\mathrm{SD}$. Droplet fusions and FRAP experiments were performed over five times with reproducible results.

\section{Extended Data Fig. 14 | ELF3 expression in the transgenic lines used in this study}

Please note that all transgenic plants used in this study were generated by expressing ELF3 gene under the control of its native promoter in the elf3-1 
mutant backgrounds. Phenotypes of the elf3-1 mutant were perfectly rescued in all ELF3 transgenic lines used in this study. Transcript levels of ELF3 gene were determined by RT-qPCR. Gene expression values were normalized to the elF4A expression. Biological triplicates were averaged. Error bars indicate standard error of the mean. a, ELF3pro::ELF3 elf3-1 transgenic plants without any tag sequences. They were used for hypocotyl elongation and RNA-seq experiments. b, ELF3pro::ELF3-FLAG elf3-1 lines were used for flowering time measurements and ChIP-seq experiments . c, ELF3pro::ELF3-GFP elf31 lines were used for observation of ELF3 speckle formation in planta.

\section{METHODS ONLINE}

\section{Generation of transgenic plants used in study}

Arabidopsis thaliana lines used in this study were in Columbia (Col-0) background. The elf3-1, elf4-2, and elf4-101 mutants have been described previously ${ }^{2-4}$. To generate transgenic plants overexpressing ELF4 gene (ELF4-OE), the ELF4 coding sequence was subcloned into pENTR-D-TOPO vector (ThermoFisher Scientific, Rockford, IL) according to the manufacturer's procedure. The resultant entry plasmid was recombined with LR clonase into the gateway binary pJHA212B vector containing the $35 \mathrm{~S}$ promoter and Cterminal $3 x f l a g$ tag sequences. The binary construct was transformed into Col0 plants by floral dipping method. The ELF4-OE transgenic plants were isolated by basta selection, and propagated to obtain single insertion lines with phenotypes of short hypocotyls and delayed flowering. The ELF3-OE transgenic plant has been described previously ${ }^{4}$. The ELF3-OE and ELF4-OE plants were crossed with elf4-2 and elf3-1, respectively and the resultant homozygous generations were used for measurements of hypocotyl length and flowering time.

To investigate if the length of the polyglutamine (polyQ) repeat influences ELF3 activity, a $7.8 \mathrm{~kb}$ genomic fragment of ELF3 including its promoter and stop codon was firstly subcloned into $p E N T R-D-T O P O$ vector, as described above. Please note that the ELF3 protein in Col-0 plant has the 
poly $Q$ repeat sequence of $Q 7$. The $Q 7$ repeat sequence in the entry plasmid was deleted or extended to Q21 by an overlapping PCR strategy. Three kinds of entry plasmids encoding ELF3 proteins with Q0, Q7, and Q21, respectively, were was recombined with LR clonase into the gateway binary pJHA212K vector without any tagging sequences. The binary construct was transformed into the elf3-1 mutant by floral dipping method. Three kinds of transgenic plants were isolated by kanamycin selection and propagated to obtain single insertion lines rescuing the long hypocotyl phenotype in the elf3-1. The resultant homozygous generations were used for hypocotyl length measurements.

To investigate if the prion domain $(\mathrm{PrD})$ in the ELF3 protein confers thermal responsiveness, we generated transgenic plants expressing StELF3 and BdELF3 under the control of both the native Arabidopsis ELF3 and $35 \mathrm{~S}$ promoters in elf3-1. Genomic DNA was first isolated from the nuclei of Solanum tuberosum and Brachypodium distachyon using a standard CTAB DNA extraction method. Coding sequences of StELF3 and BdELF3 genes were amplified by PCR using the genomic DNA from Solanum and Brachypodium, respectively, as template. The PCR fragments were cloned into the SLIC binary vector containing the $35 \mathrm{~S}$ promoter and N-terminal 3xflag tag sequences using NEBuilder ${ }^{2}$ HiFi DNA Assembly Cloning Kit (New England BioLabs, Hertfordshire, UK), and the constructs were transformed into the elf3-1 mutant, resulting in StELF3-OE and BdELF3-OE, respectively. The same PCR fragments were also cloned into the SLIC binary vector containing the Arabidopsis ELF3 promoter and C-terminal 3xflag tag sequences, and the constructs were transformed into the elf3-1 mutant, resulting in ELF3pro:StELF3 and ELF3pro:BdELF3 transgenic plants, respectively. To create a chimeric version of Arabidopsis ELF3, where its $\operatorname{PrD}$ was replaced with the corresponding sequence of BdELF3, the existing entry plasmid containing the Arabidopsis ELF3 promoter and coding region was modified to replace the DNA fragment encoding PrD sequence (residues 430609aa) with the corresponding DNA fragment of BdELF3 gene (Expended Data Fig. 2). The modified entry plasmid was recombined with LR clonase into the gateway binary pJHA212K vector containing the C-terminal 3xflag tag sequence, and the constructs were transformed into the elf3-1 mutant, 
resulting in the ELF3pro:ELF3-BdPrD transgenic plant. All transgenic plants containing DNA fragments from Solanum or Brachypodium were isolated by kanamycin selection and propagated to obtain single insertion lines rescuing the long hypocotyl phenotype in the elf3-1.

Different kinds of ELF3 entry plasmids encoding Arabidopsis ELF3 proteins with the variation of polyQ length (Q0 Q35) or Brachypodium ELF3 protein were recombined into the gateway binary pJHA212K vectors containing the C-terminal 3xflag or GFP tag sequences. The resultant constructs were transformed into the elf3-1 mutant for generating transgenic plants used for chromatin immunoprecipitation sequencing (ChIP-seq) or plant fluorescence microscopy experiments, respectively. The ELF3pro:ELF3-MYC elf3-1 transgenic plant used for ChIP-seq experiments has been described previously ${ }^{3}$.

\section{Plant growth conditions}

Arabidopsis seeds were sterilized and sown on standard half-strength Murashige and Skoog-agar (MS-agar) plates at pH 5.7. Sterilized seeds were stratified for 3 days at $4{ }^{\circ} \mathrm{C}$ in the dark and allowed to germinate for 24 hours at $22{ }^{\circ} \mathrm{C}$ under cool-white fluorescent light at $170 \mu \mathrm{mol} \mathrm{m} \mathrm{m}^{-2} \mathrm{~s}^{-1}$. The plates were then transferred to short photoperiod conditions (SDs, 8-h light and 16-h dark) at different temperatures for assays. For hypocotyl length measurement, 7- or 8-day-old seedlings, which were grown under SDs with light intensity of $80 \mu \mathrm{mol} \mathrm{m} \mathrm{m}^{-2} \mathrm{~s}^{-1}$, were photographed and analyzed using ImageJ software (http://rsbweb.nih.gov/ij/).

For flowering time measurement, plants were grown in soil under SDs at either 22 or $27^{\circ} \mathrm{C}$ until flowering. Flowering times were determined by counting the number of rosette and cauline leaves at bolting. Twenty to thirty plants were counted and averaged for each measurement.

\section{ChIP-seq experiments}

Seedlings were grown for 10 days under SDs at either 17 or $22^{\circ} \mathrm{C}$ and shifted to $27^{\circ} \mathrm{C}$ for $2 \mathrm{~h}$ at ZT8. $3 \mathrm{~g}$ of seedlings for each treatment were fixed under vacuum for $20 \mathrm{~min}$ in $1 \times \mathrm{PBS}\left(10 \mathrm{mM} \mathrm{PO}_{4}{ }^{3-}, 137 \mathrm{mM} \mathrm{NaCl}\right.$, and $2.7 \mathrm{mM} \mathrm{KCl}$ ) containing 1\% Formaldehyde (F8775 Sigma). The reaction was quenched by 
adding glycine to a final concentration of $62 \mathrm{mM}$. ChIP experiments were performed as described ${ }^{20}$. Anti-c-Myc agarose affinity gel antibody (Sigma, A7470), Anti-HA-Agarose (Sigma, A2095) or Anti-Flag® M2 Affinity Gel (Sigma, A2220) were used for immunoprecipitation. Sequencing libraries were prepared using TruSeq ChIP Sample Preparation Kit (Illumina, IP-2021024) or using NEBNext $\circledast$ Ultra ${ }^{\mathrm{TM}}$ II DNA Library Prep Kit (New England BioLabs) and samples were sequenced on the Illumina NextSeq 500 platform.

\section{RNA-seq experiments}

Seedlings were grown on plates for 10 days and harvested at the indicated time points. $70 \mathrm{mg}$ of seedlings were pooled per tube and total RNA was extracted using MagMAX ${ }^{\mathrm{TM}}$-96 Total RNA Isolation Kit (ThermoFisher) according to the manufacturer's instruction. Libraries were prepared using Lexogen QuantSeq 3' mRNA-Seq Library Prep FWD Kit (Illumina) according to manufacturer's instruction. The libraries were sequenced on the Illumina NextSeq 500 platform.

\section{Bioinformatic analysis of ChIP-seq and RNA-seq data}

Pipeline: Quantification of gene expression and chipseq binding:

Figure: pile-up figure: Coverage values were extracted from RPKM bigwig outputs from the pipeline for [File:"chipseq_differential_binding.peak_list.csv"]. The figure is shaded with standard error computed for each $\mathrm{x}$-value.

File: "chipseq_differential_binding.peak_list.csv"

Two ELF3 ChIP-Seq libraries were compared to shortlist $3621 \mathrm{bp}$ genomic intervals that show reduced binding in $27 \mathrm{C}$ compared to $17 \mathrm{C}$. These genomic intervals are used for pileup of other ChIP-Seq libraries.

File: "chipseq_targets_genes_job.peak_list.csv"

Continuing from "chipseq_differential_binding.peak_list.csv", the genomic intervals are filtered out if it lacks an annotated start codon within 500bp. These genes are then deposited into 
File: "chipseq_differential_binding.peak_list.csv"

Column:"chipseq_targets_genes_job"

see "chipseq_targets_genes_job.peak_list.csv"

Column: "signature_targets"

A signature_score is computed for each of $\sim 36 \mathrm{k}$ annotated genes, according to their similarity to a signature gene LUX within 10 selected datasets. The top $1 \%$ genes were then selected to be "signature_targets". The signature score is defined as

$\mathrm{s}=<$ meanNorm(expression(gene)), meanNorm(expression(LUX)) $>\mathrm{s}=<$ meanNo rm(expression(gene)), meanNorm(expression(LUX))>

where $<$ expr1,expr2><expr1,expr2> is the dot product taken over the selected datasets.

\section{Analysis of gene transcript levels}

Gene transcript levels were determined by RT-qPCR. Isolation of total RNA from appropriate plant materials was carried out using Trizol reagent (Thermo Fisher Scientific) according to the manufacturer's recommendations. First strand cDNA was synthesized from $1.5 \mu \mathrm{g}$ of total RNA using RevertAid First Strand cDNA Synthesis Kit (Thermo Fisher Scientific) according to the manufacturer's recommendations. RT-qPCR reactions were performed in 96well blocks with the QuantStudio 1 Real-Time PCR System (Thermo Fisher Scientific) using the TOPreal qPCR 2XPreMIX (SYBR Green with high ROX, Enzynomics, Daejeon, Korea) in a final volume of $20 \mu \mathrm{l}$. The PCR primers used are listed in Table. The values for each set of primers were normalized relative to the EUKARYOTIC TRANSLATION INITIATION FACTOR 4A1 (elF4A) gene (At3g13920). All RT-qPCR reactions were performed in three technical replicates using total RNA samples extracted from three independent biological replicate samples.

\begin{tabular}{|l|l|l|}
\hline Gene & Forward primer & Reverse primer \\
\hline ELF3 & TCTAGTCAGCCTTGTGGTGTG & TCCTCTGATCATGCTGTGCC \\
\hline
\end{tabular}




\begin{tabular}{|l|l|l|}
\hline$F T$ & GGTGGAGAAGACCTCAGGAA & GGTTGCTAGGACTTGGAACATC \\
\hline elF4A & TGACCACACAGTCTCTGCAA & ACCAGGGAGACTTGTTGGAC \\
\hline
\end{tabular}

\section{Plant fluorescence microscopy}

Seeds were sown on MS-agar plates and stratified for two to three days at 4 ${ }^{\circ} \mathrm{C}$ in the dark. The plates were then transferred into short photoperiod conditions and grown for 7 days at $17^{\circ} \mathrm{C}$. Roots were imaged before and after incubation of the slides at $35{ }^{\circ} \mathrm{C}$ for $15 \mathrm{~min}$, or after 2 hours of incubation of the seedlings on pre-warmed MS-agar plates at 27 degrees, on a Zeiss LSM880 upright confocal microscope with a $20 \times$ dry Plan-Apochromatic 0.8 NA objective lens and acquired using ZEN 2.3 software (Carl Zeiss Ltd, Jena, Germany). GFP fluorescence was excited with a $488 \mathrm{~nm}$ line from an argon laser. Images were saved as czi files and then subsequently imported to ImageJ software.

For calculating the speckle score based on flurorescence intensity (Extended Data Fig. 9), regions of the roots corresponding to the size of individual cells were selected, and the mean, standard deviation (sd) and maximum (max) gray value were measured in ImageJ. It was assumed that speckle formation would lead to higher gray values and higher frequency of speckles within the analysis region would increase the standard deviation. A speckle score was obtained by calculating the ratio of the max gray value and the mean gray value, normalised to the average of the mean gray values for all the regions measured in each root (to account for local intensity variation), and finally multiplied by the standard deviation, according the following formula:

speckle score $=[\max ($ grey value $) /$ mean (grey value $)] /$ average $($ mean grey value for all regions in the root) $x$ sd

\section{Yeast fluorescence microscopy}

Yeast cells (RS453 MATa ade2-1 his3-11,15 ura3-52 leu2-3112 trp1-1, URA3::YIplac211-SEC63-mCherry) ${ }^{21}$ were transformed with plasmids in the table below using the lithium acetate method, and grown in synthetic defined (SD) medium containing $0.17 \%$ Yeast Nitrogen Base (MP Biomedicals, Santa 
Ana, CA), 0.5\% ammonium sulfate (Fisher Scientific, Leicestershire, UK), LEU/-TRP DO supplement (Clontech, Kusatsu, Japan), and $60 \mathrm{mg} \mathrm{l}^{-1}$ leucine or $40 \mathrm{mg} \mathrm{l}^{-1}$ tryptophan (Sigma, St. Louis, MO), for plasmid selection. Cells were grown overnight at $19{ }^{\circ} \mathrm{C}$, incubated at $35^{\circ} \mathrm{C}$ for $30 \mathrm{~min}$ and, where indicated, re-incubated at $19{ }^{\circ} \mathrm{C}$ for $60 \mathrm{~min}$. Cells were imaged live in a Zeiss Axiolmager.Z2 epifluorescence upright microscope with a 100x PlanApochromatic 1.4 NA objective lens (Carl Zeiss Ltd, Jena, Germany). Images were recorded using a large chip sCMOS mono camera for sensitive fluorescence imaging (ORCA Flash 4.0v2, Hamamatsu, Hamamatsu, Japan), saved by Zeiss ZEN2.3 software (Blue edition, Carl Zeiss Ltd, Jena, Germany) and exported to ImageJ software.

\begin{tabular}{|l|l|l|}
\hline Plasmid & Description & Source \\
\hline YCplac111- & $\begin{array}{l}\text { GFP under the control of NOP1 promoter in } \\
\text { LEU2/CEN vector }\end{array}$ & $\begin{array}{l}\text { S. } \\
\text { Siniossoglou } \\
\text { Lab }\end{array}$ \\
\hline $\begin{array}{l}\text { YCplac111- } \\
\text { NOP-cELF3- }\end{array}$ & $\begin{array}{l}\text { C-terminally GFP tagged WT ELF3 cDNA under } \\
\text { the control of NOP1 promoter in LEU2/CEN } \\
\text { vector }\end{array}$ & This study \\
\hline $\begin{array}{l}\text { YCplac111- } \\
\text { NOP-cELF3- }\end{array}$ & $\begin{array}{l}\text { C-terminally GFP tagged ELF3 cDNA with a } \\
\text { polyQ repeat containing 35 glutamines under } \\
\text { the control of NOP1 promoter in LEU2/CEN } \\
\text { vector }\end{array}$ & \\
\hline $\begin{array}{l}\text { YCplac111- } \\
\text { NOP-cELF3- }\end{array}$ & $\begin{array}{l}\text { C-terminally GFP tagged ELF3 cDNA with the } \\
\text { PrD domain of } B \text {. distachyon under the control of }\end{array}$ & This study \\
\hline $\begin{array}{l}\text { NOP1 promoter in LEU2/CEN vector } \\
\text { CELF3-Q7-GFP }\end{array}$ & $\begin{array}{l}\text { C-terminally GFP tagged WT ELF3 cDNA under } \\
\text { the control of } A D H 1 \text { promoter in TRP1/2 vector }\end{array}$ & This study \\
\hline
\end{tabular}

\section{Yeast two hybrid assays}

Yeast two-hybrid assays were performed using the BD Matchmaker system (Clontech, Palo Alto, CA). The pGADT7 vector was used for GAL4 activation domain, and the pGBKT7 vector was used for GAL4 DNA-binding domain. Clontech's $\mathrm{Y} 2 \mathrm{H}$ Gold yeast strain was used for transformation. ELF4 and ELF3 cDNA sequences were subcloned into pGBKT7 and pGADT7 vectors, respectively. Transformation of vector constructs into $\mathrm{Y} 2 \mathrm{H}$ Gold cells was 
performed according to the manufacturer's instructions. Colonies obtained were streaked on selective medium without Leu, Trp, His, and Ade (-LWHA).

\section{ELF3 PrD constructs}

Arabidopsis ELF3 PrD (residues 388-625, AT2G25930) and Brachypodium ELF3 PrD (residues 432-669, BRADI_2g14290) were cloned into the expression vector pESPRIT2 $2^{22,23}$ using the Aat II and Not I sites. The plasmid contains an $\mathrm{N}$-terminal 6 -His tag followed by a TEV protease cleavage site. All proteins were overproduced in E. coli BL21 Rosetta 2 (Novagen).

\section{Protein Expression and purification}

BdELF3 PrD, AtELF3 PrD and AtELF3 PrD-GFP were expressed in Escherichia coli BL21, which were induced with $1 \mathrm{mM}$ IPTG (isopropyl- $\beta-d-$ thiogalactopyranoside), at $18^{\circ} \mathrm{C}$ overnight. Bacterial pellets were resuspended in resuspension buffer (100 mM CAPS pH 9.7, $300 \mathrm{mM} \mathrm{NaCl}, 30 \mathrm{mM}$ Imidazole, $1 \mathrm{mM}$ TCEP (tris(2-carboxyethyl)phosphine, Sigma) plus cOmplete protease inhibitor cocktail (Roche). Cells were lysed by sonication and the lysates were centrifuged at $50,000 \times \mathrm{g}$ for $30 \mathrm{~min}$ at $4^{\circ} \mathrm{C}$. For AtELF3 PrD and AtELF3 PrD-GFP, the supernatants were applied to a Ni-NTA column. The bound proteins were washed with $20 \mathrm{CV}$ of resuspension buffer and then with $20 \mathrm{CV}$ of a high salt buffer (100 mM CAPS pH 9.7, $1 \mathrm{M} \mathrm{NaCl}, 30 \mathrm{mM}$ imidazole and $1 \mathrm{mM}$ TCEP) and eluted with $5 \mathrm{CV}$ of elution buffer (100 mM CAPS pH 9.7, $300 \mathrm{mM} \mathrm{NaCl}, 300 \mathrm{mM}$ imidazole and $1 \mathrm{mM}$ TCEP). The fractions of interest were pooled and dialyzed overnight at $4{ }^{\circ} \mathrm{C}$ in $50 \mathrm{mM}$ CAPS $\mathrm{pH}$ 9.7, $400 \mathrm{mM} \quad \mathrm{NaCl}$ and $1 \mathrm{mM}$ TCEP. For BdELF3 PrD, the pellet was solubilised in $8 \mathrm{M}$ urea, $100 \mathrm{mM}$ CAPS pH 9.7 and $300 \mathrm{mM} \mathrm{NaCl}$. A second centrifugation was performed and the supernatant was applied to a Ni-NTA column pre-equilibrated with equilibration buffer (8 M urea, 100 mM CAPS pH 9.7, 300 mM NaCl, 30 mM imidazole and $1 \mathrm{mM}$ TCEP). The bound protein was washed with $20 \mathrm{CV}$ of equilibration buffer and then with $20 \mathrm{CV}$ high salt buffer for on-column refolding. The protein was eluted with $5 \mathrm{CV}$ of elution buffer. Fractions of interest were pooled and dialyzed overnight at $4{ }^{\circ} \mathrm{C}$ in $50 \mathrm{mM} \mathrm{CAPS} \mathrm{pH} \mathrm{9.7,}$ 
$300 \mathrm{mM} \mathrm{NaCl}$ and $1 \mathrm{mM}$ TCEP. Protein purity was determined via SDSPAGE.

\section{Liquid droplet formation}

To form liquid droplets, the $\mathrm{NaCl}$ concentration of the dialysis buffer (50 mM CAPS $\mathrm{pH} 9.7,400 \mathrm{mM} \mathrm{NaCl}$ and $1 \mathrm{mM}$ TCEP) was gradually decreased using a step gradient at $4{ }^{\circ} \mathrm{C}$. Droplets were visualized after dialysis. Images were acquired at the 20X objective (LUCPLFLN20xPH1/0.45) on an epifluorescence inverted microscope (CKX41 model) equipped with a pE-300 Cool-LED camera.

\section{AtELF3PrD-GFP FRAP}

For droplet visualization and photobleaching experiments of AtELF3PrD-GFP protein, liquid droplet formation was induced by mixing $5 \mu \mathrm{L}$ of $5 \mathrm{mg} / \mathrm{mL}$ AtELF3PrD-GFP with $5 \mu \mathrm{L} 20 \mathrm{mM}$ Tris $\mathrm{pH}$ 7.5, $100 \mathrm{mM} \mathrm{NaCl}, 1 \mathrm{mM}$ TCEP on a glass slide. The drop was covered with a cover slip and quickly mounted onto the EclipseTi-E Nikon inverted microscope as part of the confocal spinning disk system with a CSUX1-A1 Yokogawa confocal head, an Evolve EMCCD camera (Roper Scientific, Princeton Instruments) and a Nikon CFI Plan-APO VC 60x, 1.4 N.A, oil-immersion objective controlled with the MetaMorph (Universal Imaging) software with the autofocus function enabled. For photobleaching experiments, droplets were allowed to adhere to the coverslip prior to photobleaching to minimize droplet movement during the experiment. Acquisition times were approximately $1 \mathrm{~s}$ per image. Droplet size was $\sim 2-5 \mu \mathrm{m}$ with bleaching area of $\sim 1 \mu \mathrm{m}$ for partial bleaching and $\sim 5 \mu \mathrm{m}$ for full bleaching. Time-lapse images were acquired at $530 \mathrm{nM}$. Droplet intensity profiles were measured manually for droplet fusion quantification in Image ${ }^{24}$. For FRAP experiments, regions of interest (bleached, unbleached and background) were selected in ImageJ and processed with the easyFRAP webtool $^{25}$. Corrected intensities were fit to a single exponential curve in ImageJ.

\section{Light scattering assay}


The light scattering assay was performed in a Cary 100 UV-vis spectrometer (Agilent Technologies UK Ltd., Stockport, UK). The absorbance at $440 \mathrm{~nm}$ was monitored for samples containing buffer alone (50 mM CAPS pH 9.7, 150 $\mathrm{mM} \mathrm{NaCl}, 1 \mathrm{mM}$ TCEP), ELF3 PrD $(15 \mu \mathrm{M})$ or BdELF3 $(15 \mu \mathrm{M})$ in quartz cuvettes (path length $10 \mathrm{~mm}$ ) with increasing temperature $\left(4-50^{\circ} \mathrm{C} ; 1^{\circ} \mathrm{C}\right.$ min $^{-}$ ${ }^{1}$ ), and the spectra were normalized with respect to ELF3 PrD. A transition temperature $\left(T_{m}\right)$ was determined by fitting the spectrum with a 4-parameter sigmoidal equation (Sigmaplot 11, Systat Software Inc.). Reported values are an average of three separate experiments. To monitor reversibility, the turbidity was monitored whilst increasing temperature $\left(10\right.$ to $\left.40^{\circ} \mathrm{C} ; 1^{\circ} \mathrm{C} \mathrm{min}{ }^{-1}\right)$ followed by decreasing the temperature $\left(40\right.$ to $10^{\circ} \mathrm{C} 1^{\circ} \mathrm{C} \mathrm{min}{ }^{-1}$ ) and this cycle was repeated three times in total (ELF3 PrD (5 or 15, $\mu \mathrm{M}), 50 \mathrm{mM} \mathrm{CAPS} \mathrm{pH}$ 9.7, $200 \mathrm{mM} \mathrm{NaCl}, 1 \mathrm{mM}$ TCEP).

20. Jaeger, K. E., Pullen, N., Lamzin, S., Morris, R. J., \& Wigge, P. A. Interlocking feedback loops govern the dynamic behavior of the floral transition in Arabidopsis. Plant Cell 25, 820-833 (2013).

21. Barbosa, A. D. et al. Lipid partitioning at the nuclear envelope controls membrane biogenesis. Mol. Biol. Cell 26, 3641-3657 (2015).

22. Guilligay, D. et al. The structural basis for cap binding by influenza virus polymerase subunit PB2. Nat. Struct. Mol. Biol. 15, 500-506 (2008).

23. Tarendeau, F. et al. Structure and nuclear import function of the Cterminal domain of influenza virus polymerase PB2 subunit. Nat. Struct. Mol. Biol. 14, 229-233 (2007).

24. Schindelin, J.; Arganda-Carreras, I. \& Frise, E. et al. Fiji: an opensource platform for biological-image analysis. Nature Methods 9, 676682 (2012).

25. Koulouras G, Panagopoulos A, Rapsomaniki MA, Giakoumakis NN, Taraviras S, Lygerou Z EasyFRAP-web: a web-based tool for the analysis of fluorescence recovery after photobleaching data, Nucleic Acids Res 46, W467-W472 (2018). 


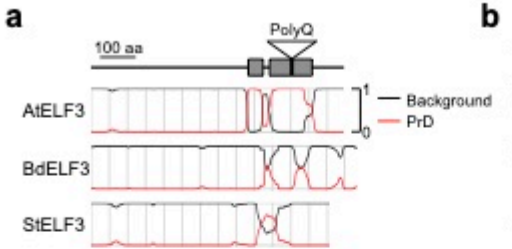

C

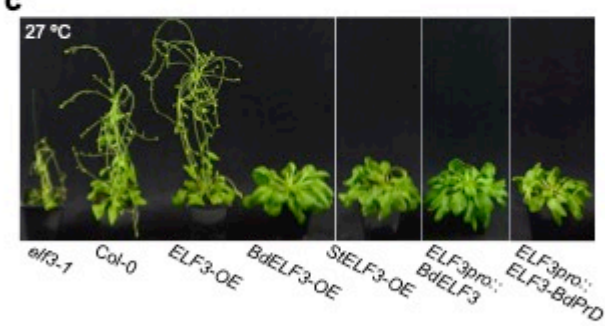

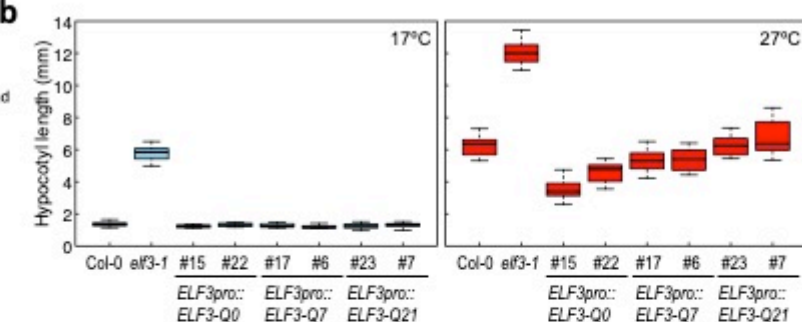
ELF3-Q0 ELF3-Q7 ELF3-Q21

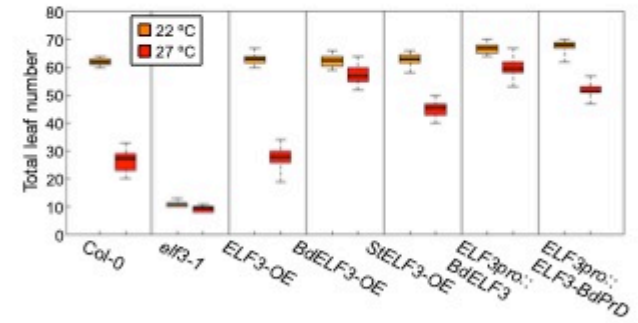

Fig. 1: The polyQ repeat of ELF3 is embedded within a predicted priondomain which is essential for thermal responsiveness.

a, Arabidopsis ELF3 (AtELF3) contains a polyQ repeat embedded with a predicted prion-domain ( $\operatorname{PrD}$ ), while this PrD signature is absent in ELF3 from Brachypodium distachyon (BdELF3) and Solanum tuberosum (SdELF3). Prion domains predicted using PLAAC ${ }^{12}$. b, At $17^{\circ} \mathrm{C}, E L F 3$ is required to prevent hypocotyl elongation, but different polyQ lengths in ELF3 do not perturb ELF3 function. At $27^{\circ} \mathrm{C}$, the responsiveness of ELF3 to temperature increases with the length of the polyQ. (Naming convention: Q7-17, 7 refers to length of polyQ repeat, and 17 is the individual transgenic line used in the study). c, The ELF3 PrD plays a critical role in the thermal control of flowering time. Overexpressing AtELF3 does not change the thermal induction of flowering, indicating that simply increasing ELF3 protein level is not sufficient to delay flowering, while overexpressing BdELF3 causes an almost complete loss of thermal induction, and StELF3 has a milder influence. These effects are also apparent when $B d E L F 3$ is expressed using the native ELF3 promoter, and dependent on the $\operatorname{PrD}$, since replacing this domain of AtELF3 with the corresponding Bd sequence is sufficient to greatly reduce the thermal induction of flowering. 

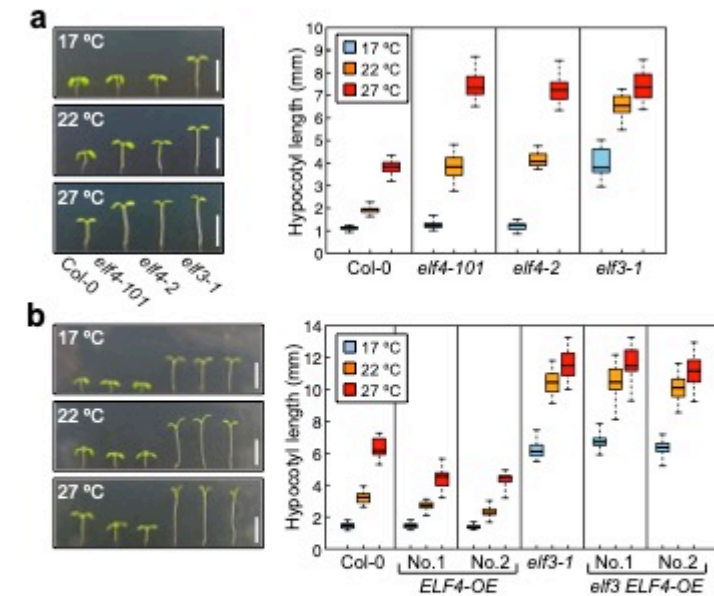

C

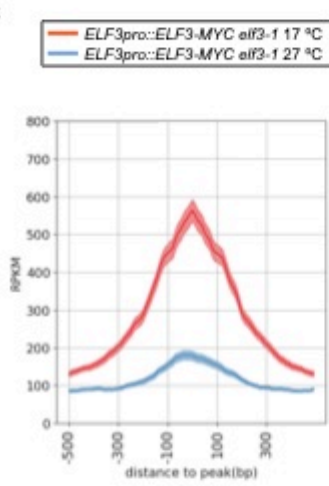

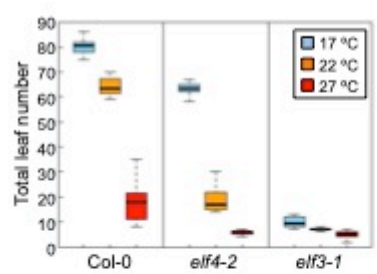
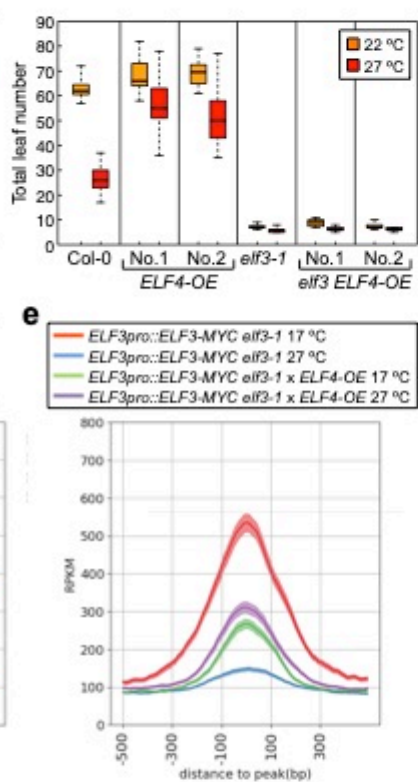

Fig. 2: ELF4 is required to stabilize the activity of the EC at warmer temperatures.

a, At lower temperatures, ELF4 becomes dispensable for controlling both hypocotyl elongation and flowering, but with increasing temperature, ELF4 assumes a greater role. b, ELF4 overexpression greatly reduces thermal responsiveness of both hypocotyl elongation and flowering time, and this response is entirely dependent on ELF3. c, ELF3 binding at targets, which is measured by ChIP-seq, is temperature dependent and declines genome-wide at $27^{\circ} \mathrm{C}$. d, Transgenic plants with stabilized forms of ELF3 do not respond to temperature. e, ELF4 overexpression stabilizes ELF3 and removes its temperature responsiveness of binding to targets. Scale bars, $5 \mathrm{~mm}(\mathbf{a}, \mathbf{b})$ 

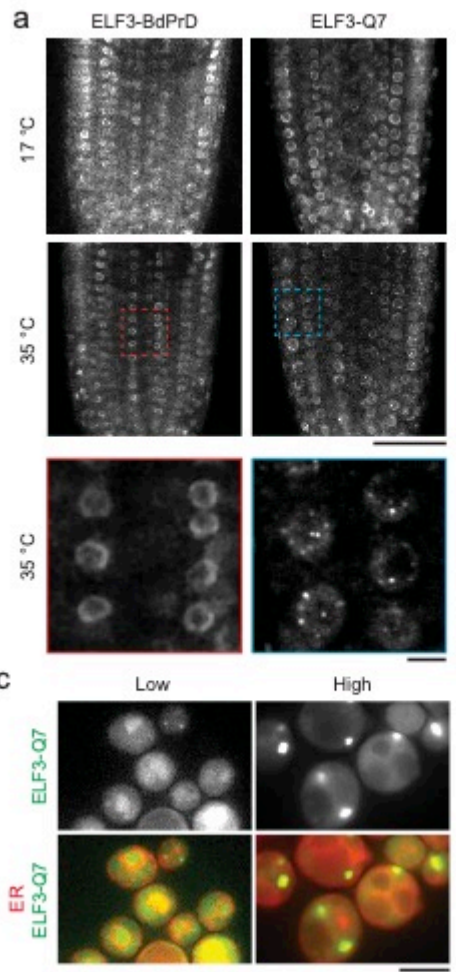

b
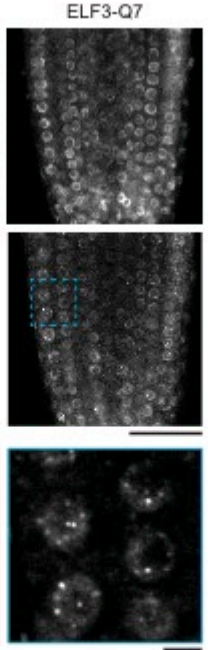

igh .

d

e
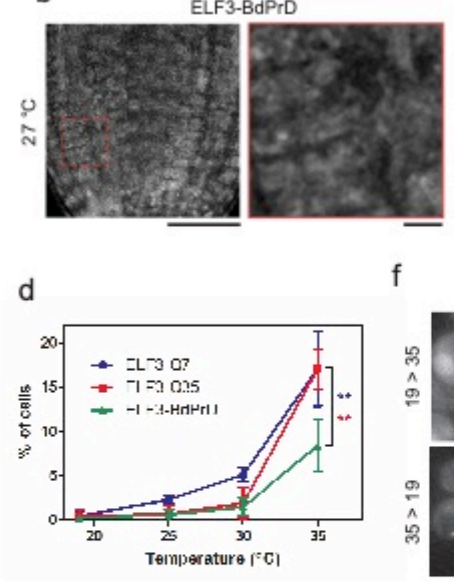

f
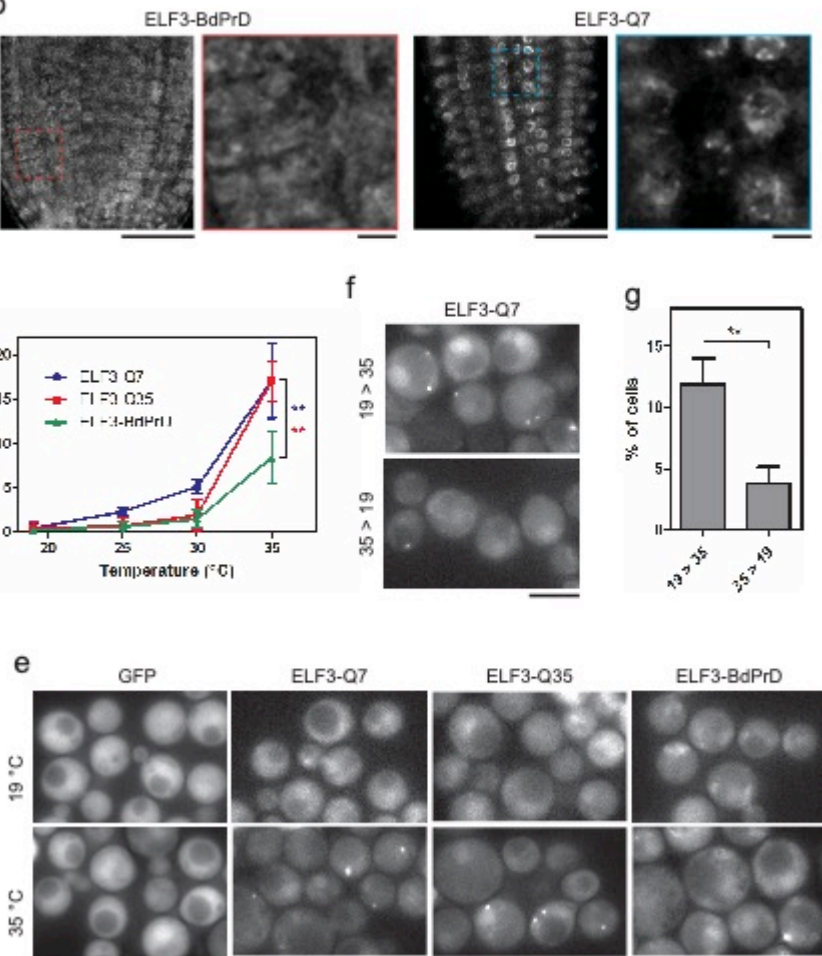

g

ELF3-Q7

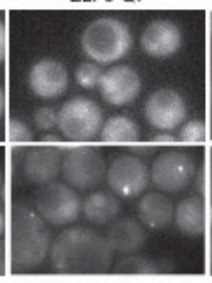

ELF3-Q35

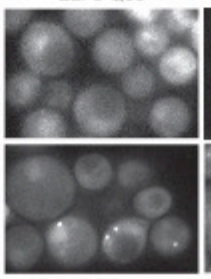

ELF3-BdPrD

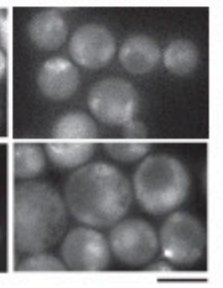

Fig. 3: High temperature induces the formation of ELF3 speckles in vivo.

a, Seedlings expressing GFP-tagged ELF3 (Q7) or a chimeric ELF3 where the PrD has been replaced by the corresponding region of Brachypodium ELF3 (BdPrD) grown in short photoperiods for 7 days at $17^{\circ} \mathrm{C}$. Roots imaged by confocal microscopy before and after incubation at $35^{\circ} \mathrm{C}$ for $15 \mathrm{~min}$. b. Seedlings as in a but shifted to $27^{\circ} \mathrm{C}$ for 2 hours. c, S. cerevisiae cells expressing GFP-tagged ELF3 (Q7) from a centromeric plasmid (Low) or an episomal plasmid (High), to achieve low or high expression levels of the protein, were grown in selective synthetic complete medium at $30^{\circ} \mathrm{C}$. Sec63-mCherry was used as an ER reporter. d, Quantification of ELF3 speckles in S. cerevisiae cells expressing free GFP (GFP only), ELF3 (Q7), ELF3 with a longer polyQ repeat (Q35), or ELF3 with the PrD of Brachypodium (BdPrD) were grown overnight at $19{ }^{\circ} \mathrm{C}$ and incubated at the indicated temperatures for $30 \mathrm{~min}$. e, Representative images of cells from $\mathbf{d}$ at $35^{\circ} \mathrm{C}$. f, S. cerevisiae cells expressing ELF3 were grown at $19{ }^{\circ} \mathrm{C}$ overnight and incubated at $35^{\circ} \mathrm{C}$ for $30 \mathrm{~min}(19>35)$ followed by the incubation at $19^{\circ} \mathrm{C}$ for $60 \mathrm{~min}(35>19)$. Cells shifted from 35 to $19^{\circ} \mathrm{C}$ show a loss of speckles. g, Quantification of ELF3 speckles for cells in $\mathbf{f}$. Scale bars, $40 \mu \mathrm{m}$ (a and b) and 5 $\mu \mathrm{m}$ (a and b inset, $\mathbf{c}, \mathbf{e}$, and f), ${ }^{\star \star} \mathrm{p}<0.01$. 


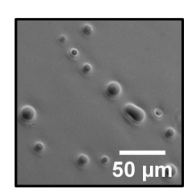

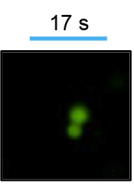
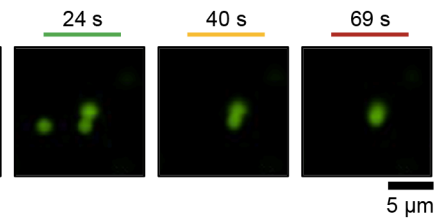

d
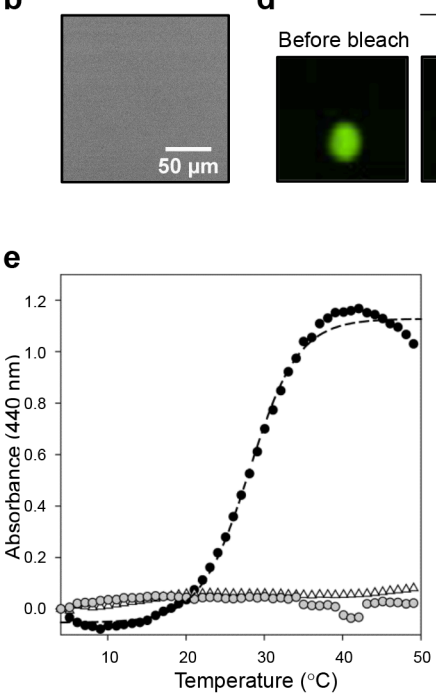

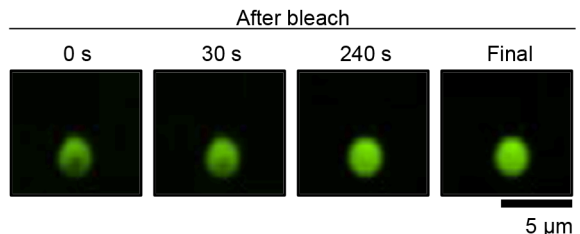

f

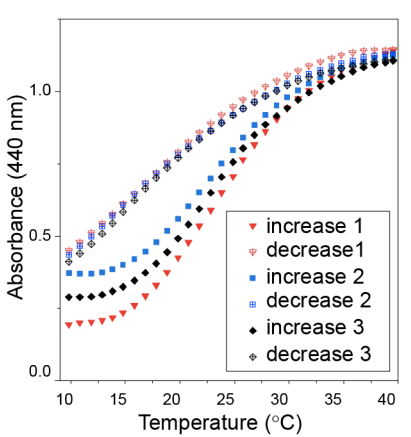

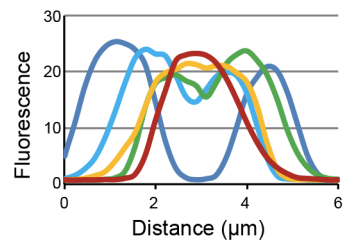

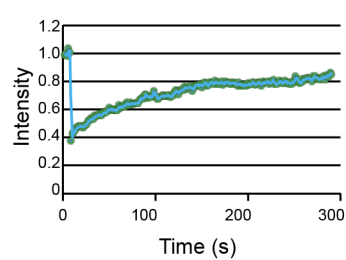

Fig. 4: The PrD of ELF3 undergoes a reversible phase transition in response to temperature.

a, Purified ELF3 PrD peptide forms liquid droplets at $27^{\circ} \mathrm{C}$ in vitro. b. The equivalent protein domain from BdELF3, which is not predicted to contain a PrD, remains soluble and does not show any liquid droplet formation c, Purified ELF3 PrD-GFP protein forms spherical droplets in vitro, which fuse. d, ELF3 PrD-GFP droplets show rapid recovery after photobleaching, indicating they are liquid droplets. e, Light scattering assay as a function of temperature for ELF3 PrD (15 $\mu \mathrm{M}$; black circle), BdELF3 (15 $\mu \mathrm{M}$; grey circle) and buffer alone (50 mM CAPS $\mathrm{pH}$ 9.7, $150 \mathrm{mM} \mathrm{NaCl}, 1 \mathrm{mM}$ TCEP; open triangles). Dashed line shows curve-fitting using a 4parameter sigmoidal equation. The $T_{m}$ for ELF3 $\operatorname{PrD}$ is $28.7 \pm 1.8^{\circ} \mathrm{C}$ and the spectrum is representative of three independent experiments. $f$, Reversibility of light scattering as a function of temperature for ELF3 PrD (15 $\mu \mathrm{M} ; 50 \mathrm{mM}$ CAPS pH 9.7, $200 \mathrm{mM} \mathrm{NaCl}, 1 \mathrm{mM}$ TCEP). On the same sample, the temperature was increased and decreased three times in succession $\left(1^{\circ} \mathrm{C} / \mathrm{min}\right)$. The observed turbidity was reversible and consistently returned to $\mathrm{Abs}_{440 \mathrm{~nm}}=0.432 \pm 0.02$. Interestingly, the initial absorbance reading for repeat $3\left(\mathrm{Abs}_{440 \mathrm{~nm}}=\right.$ $0.288)$ is lower than for repeat $2\left(\mathrm{Abs}_{440 \mathrm{~nm}}=0.373\right)$ and this is likely due time dependent equilibration (as noted in Extended Figure 12c). The spectra are representative of two independent experiments and similar results were observed for samples at $5 \mu \mathrm{M}$. Scale bars, $50 \mu \mathrm{m}(\mathbf{a}, \mathbf{b})$ and $5 \mu \mathrm{m}(\mathbf{c}, \mathbf{d})$. 